REVISTA ECONOMÍA

Vol. 69, N. ${ }^{\circ}$ I09 (mayo), I79-188

\title{
LA CALIDAD DE VIVIENDA EN LA PROVINCIA DE TUNGURAHUA: ANÁLISIS DE HETEROGENEIDAD ESPACIAL A TRAVÉS DEL MODELO SANOVA Y ANOVA
}

\author{
JULIO VILLA, ÁLVARO VAYAS, PAULINA CABAY \\ Universidad Técnica de Ambato
}

Recepción manuscrito: 6 de enero de 2017

Aceptación versión final: 15 de abril de 2017

\begin{abstract}
RESUMEN El trabajo describe la distancia de los asentamientos urbanos a la cabecera cantonal de la provincia de Tungurahua (Ecuador), explica la calidad de vivienda de la población, proponiéndose la hipótesis de que mientras más distante se encuentre un asentamiento urbano de la principal urbe provincial, la calidad de la vivienda disminuye. Se explica la relación entre la calidad de vida y la presencia de obras públicas — agua potable y alcantarillado-. Para el primer caso se utiliza un modelo de análisis de varianzas espacial —SANOVA-, la segunda relación se trata de explicar a través de un modelo ANOvA no espacial.
\end{abstract}

PALABRAS CLAVE Modelo SANOvA, modelo ANOva, calidad de vivienda.

ABSTRACT The paper describes the distance from urban settlements to the cantonal headland of the province of Tungurahua (Ecuador), explains the quality of housing of the population, proposing the hypothesis that the more distant an urban settlement of the main provincial city, the quality of housing decreases. The relationship between the quality of life and the presence of public works - drinking water and sewage - is explained. For the first case a model of spatial variances analysis - SANOVA - is used, the second relation is to explain through a nonspatial ANOVA model.

KEYWORDS SANOVA model, ANOVA model, housing quality.

JEL CODE C, C2, C21.

\section{INTRODUCCIÓN}

El crecimiento de las ciudades a través de la historia se ha fundamentado en las oportunidades que ofrece la ubicación de estos centros urbanos; por otro lado, estas localidades se desarrollan por la obra pública, si existe un limitado gasto público en mejoras de la infraestructura, los asentamientos se ven limitados en su desarrollo, urbanísticamente hablando. Las necesidades humanas crecen conforme el desarrollo de las generaciones, y la logística pública debe mejorarse para poder satisfacer la demanda cada vez creciente de los asentamientos urbanos, la construcción de obra pública dinamiza la transformación de las viviendas y la calidad de vida de dichos asentamientos. 
El objetivo de este análisis es comprender, por una parte, cómo se relacionan la ubicación de las residencias y la calidad de la vivienda, por otra parte, se analiza la calidad de vida expresada estrictamente a través del desarrollo urbanístico basado en la inversión pública. Se plantea el uso de un procedimiento técnico econométrico que detalle el comportamiento de las variables, para ello se utilizará un modelo análisis de varianzas espacial (sANOva) para el estudio de la relación entre la ubicación de residencia y la calidad de vivienda de las poblaciones de ocho cantones de la provincia de Tungurahua. Posteriormente, a través de un modelo no espacial (ANOVA) se buscará analizar la relación entre la obra pública y el mejoramiento de la calidad de vida de los asentamientos urbanos de la provincia.

El presente estudio se encuentra estructurado en cuatro secciones. La primera contiene el marco teórico en el cual se describen las bases conceptuales así como los antecedentes de estudio que se han realizado en torno al tema de análisis. En la sección dos se muestran los procedimientos técnicos econométricos y metodológicos que se efectuaron para la recolección de la información y el análisis econométrico de los datos empíricos observados. En la sección número tres se describen los resultados del análisis ANOva propuesto para el estudio del presente análisis con sus respectivas interpretaciones. Finalmente, en la sección cuatro se detallan las conclusiones del planteamiento de la hipótesis propuesta y del estudio de la bibliografía así como del análisis empírico realizado.

\section{ANÁLISIS GEOESPACIAL DE LA POBLACIÓN}

Para poder realizar un análisis que muestre las características espaciales, de hacinamiento o de características innatas de la ubicación de los asentamientos humanos es muy útil realizar un estudio geoespacial sectorizado que permita dirimir ciertas características de poblaciones con diferencias intrínsecas conforme a su ubicación geográfica; conocer la ubicación de un problema social en específico y su colocación en el territorio es el inicio para enfrentar la problemática y llevar a cabo las acciones necesarias a su solución. (Mena, Morales, Gajardo y Ormazábal, 2007). La utilización de encuestas así como de información de una población inmersa en una problemática relacionada a su ubicación geográfica es de mucha utilidad en especial para el análisis de la acción del Estado en el territorio; es así que Mena et al. (2007) en su estudio Análisis geoespacial de servicios básicos para las viviendas de Longaví, Retiro y Parral, Región del Maule, Chile, realiza un análisis geoespacial sectorizado que busca realizar una representación de la distribución espacial y geográfica de viviendas y la disponibilidad o falta de servicios básicos que éstas tienen; para este estudio se utilizó la información del Catastro Digital de Viviendas para la ordenación del Territorio, ejecutado por la Universidad de Talca del 2004 al 2005, y se realizaron encuestas que recolectaron información acerca de variables como el dominio de la vivienda, el hacinamiento, el material de construcción de la vivienda, las características del agua que se consume, la cantidad de habitaciones de la vivienda, la evacuación de excretas, el suministro de electricidad y la dirección referencial.

En este estudio se concluye que las políticas del gobierno han sido acertadas en cuanto a dotación de servicios básicos a las viviendas, se deduce, además, que producto de estas políticas gubernamentales las zonas urbanas tienen una mejor calidad de vida. Por lo tanto, los 
autores plantean el desafío de efectuar nuevos planes que asignen recursos para aquellos proyectos relacionados con la implementación de servicios básicos en las zonas rurales de las comunidades estudiadas, y esperan lograr una mayor equidad económica y social para los habitantes de estas localidades (Mena et al., 2007). La información recolectada permite analizar, estimar y cuantificar las necesidades de la población y la realidad en la cual se desenvuelve la economía y la calidad de vida de estos asentamientos, los cuales, además, demandan la cobertura efectiva y eficiente de servicios básicos y del mejoramiento de la infraestructura pública.

Por otro lado, la calidad de las viviendas desde la perspectiva del mercado inmobiliario se ve influenciada por una variedad de factores que estrictamente responden tanto a características intrínsecas de este mercado como al contexto económico y urbanístico de los asentamientos urbanos y rurales, haciendo que éstos adquieran una plusvalía. A través del estudio de los aspectos socioeconómicos, ambientales y de accesibilidad, es posible identificar cuál de todos estos factores tienen un mayor impacto en el valor del suelo y de qué manera se producen plusvalías o minusvalías ante ciertas acciones, como la planeación urbana, el equipamiento, la infraestructura y otras (Fitch, Soto y Garza, 2013). Estos autores en su análisis Valuación de la calidad urbano-ambiental. Una modelación hedónica: San Nicolás de los Garza, México, tratan de modelar econométricamente por medio del método de precios hedónicos (MPH), la calidad medioambiental en relación con el valor del suelo de San Nicolás de la Garza.

A través de una aproximación teórica sobre la estructuración del valor inmobiliario, se consideran las características del mercado de este sector y las teorías de su organización espacial. Se realiza una estimación econométrica de un grupo de variables que describen el comportamiento de los precios hedónicos de las viviendas, así como se muestra el excedente del consumidor para cada una de las variables independientes de la regresión planteada. Concluyen, en primera instancia, que la investigación podría ayudar al establecimiento de políticas en cuanto a términos de impuesto predial hasta la provisión de bienes públicos, por lo que los planes de los gobiernos locales deberían prestar mayor atención a la localización de actividades, al transporte público y a los espacios abiertos.

A través del estudio, también se logró establecer que a partir de la invasión de las clases media y media-alta de zonas que habitualmente ocupaba la clase baja se intensifican las desigualdades sociales en escala reducida, se establece también que los barrios ubicados en donde hay más riqueza acceden a mejores niveles de educación, a mejores relaciones sociales y a mejores oportunidades para conseguir buenas fuentes de trabajo (Fitch et al. 2013).

La ubicación espacial y geográfica de las localidades tiene relación con la calidad de vivienda, así como la infraestructura de obra pública incide en la calidad de vida, esto implica que la ubicación geográfica o espacial y la obra pública de los asentamientos influyen en el desarrollo socioeconómico de la población. El análisis econométrico de los aspectos particulares de una población de acuerdo a las características intrínsecas de su ubicación geográfica, constituye un tema de estudio muy importante para el análisis de las diferencias existentes en ciertos sectores de la población. La heterogeneidad espacial se presenta cuando se trabaja con observaciones de características espaciales, esta circunstancia específica del análisis de las características económicas y sociales de muestras ubicadas ya no a través del tiempo, sino en el contexto espacial, amerita un trato de la metodología econométrica específico que permita 
dirimir entre los efectos de encontrarse ubicado en un lugar o espacio determinado con respecto a otro. El efecto de heterogeneidad espacial puede ser tratado a través de técnicas econométricas tradicionales, para tal efecto existe la posibilidad de aplicar la metodología ANOvA con el objeto de poder explicar este tipo de comportamientos económicos o sociales. El tratamiento de relaciones de respuesta cuantitativa de estímulo cualitativo o dicótomo se basa en el desarrollo de un modelo de regresión ANOva, «el análisis espacial de la varianza (sAnOva) mide el grado de las diferencias por grupos o zonas del espacio» (Chasco, 2001), que resulta la metodología idónea para contrastar la hipótesis propuesta en este artículo.

Una característica específica de este tipo de modelos econométricos es el tratamiento de la variable de bloque, que si ésta es de características cualitativas tiene que ser expresada en términos dicótomos, obviando una variable que será representada por el intercepto. Un punto muy importante a tomar en cuenta son las estrategias que adopta el Estado para dinamizar aquellos asentamientos urbanos que se encuentran en las periferias o que simplemente se han quedado relegados en el desarrollo económico y urbanístico. Conforme crecen las urbes o los asentamientos cercanos, el dinamismo en cuanto al desarrollo socioeconómico se ve incrementado, por tanto, si los gobiernos locales hacen obra pública, la plusvalía de las viviendas crece debido al incremento del valor del suelo.

En el estudio La renovación como estrategia de desarrollo urbano en Buenos Aires (19962011), se analizan las estrategias adoptadas por el gobierno de la ciudad para la mejora y la renovación urbana de los barrios del sureste: Barracas, San Telmo, La Boca y Parque Patricios, en tal estudio se observó una clara inversión en espacio público e infraestructura del gobierno local, lo que incentivó a los barrios al desarrollo de infraestructura como en cuanto a calidad de vida. En este estudio se plantea analizar las estrategias que promovieron la renovación en lo que refiere a infraestructura y calidad de vida de los barrios de la ciudad de Buenos Aires, así como a través de la descripción de una matriz de políticas urbanas se intenta describir tanto aciertos como desaciertos en cuanto a la acción estatal de obras de infraestructura y en el ámbito turístico y cultural (Guevara, 2013).

Por otra parte, una manera de fomentar el desarrollo equitativo de las periferias y de las localidades urbanas podría basarse en el establecimiento de un fondo especial que vaya dirigido al crecimiento económico como urbanístico de estos sectores. Se plantea la creación de un Fondo de Compensación Regional (FCR), que comprometa a través de una preasignación fiscal la inversión del Estado y de los gobiernos locales en obra pública de las periferias colombianas y asegurar el desarrollo de estos sectores (Galvis y Meissel, 2009). La propuesta del FCR se basa en una estrategia global que supone tres pilares que son: el fondo de compensación regional, estrategia de desarrollo productivo y nueva ética de lo público; en las cuales se encaminan acciones de lograr mayores niveles de bienestar poblacional, mantener la equidad económica y social así como la igualdad de oportunidades para todas las regiones de Colombia.

Los resultados del análisis empírico reportan que la zona norte, que comprende la región Caribe y norte de Santander, es la que demanda atención de recursos para reducir las brechas sociales y económicas y atender las necesidades de sus ciudadanos. También se encuentra que la periferia del país es la que peores indicadores socioeconómicos presenta. Por lo que se postula que el FCR sería la política pública más acertada para impulsar ese gran empuje económico 
Tabla 1. Preguntas consideradas para el análisis econométrico

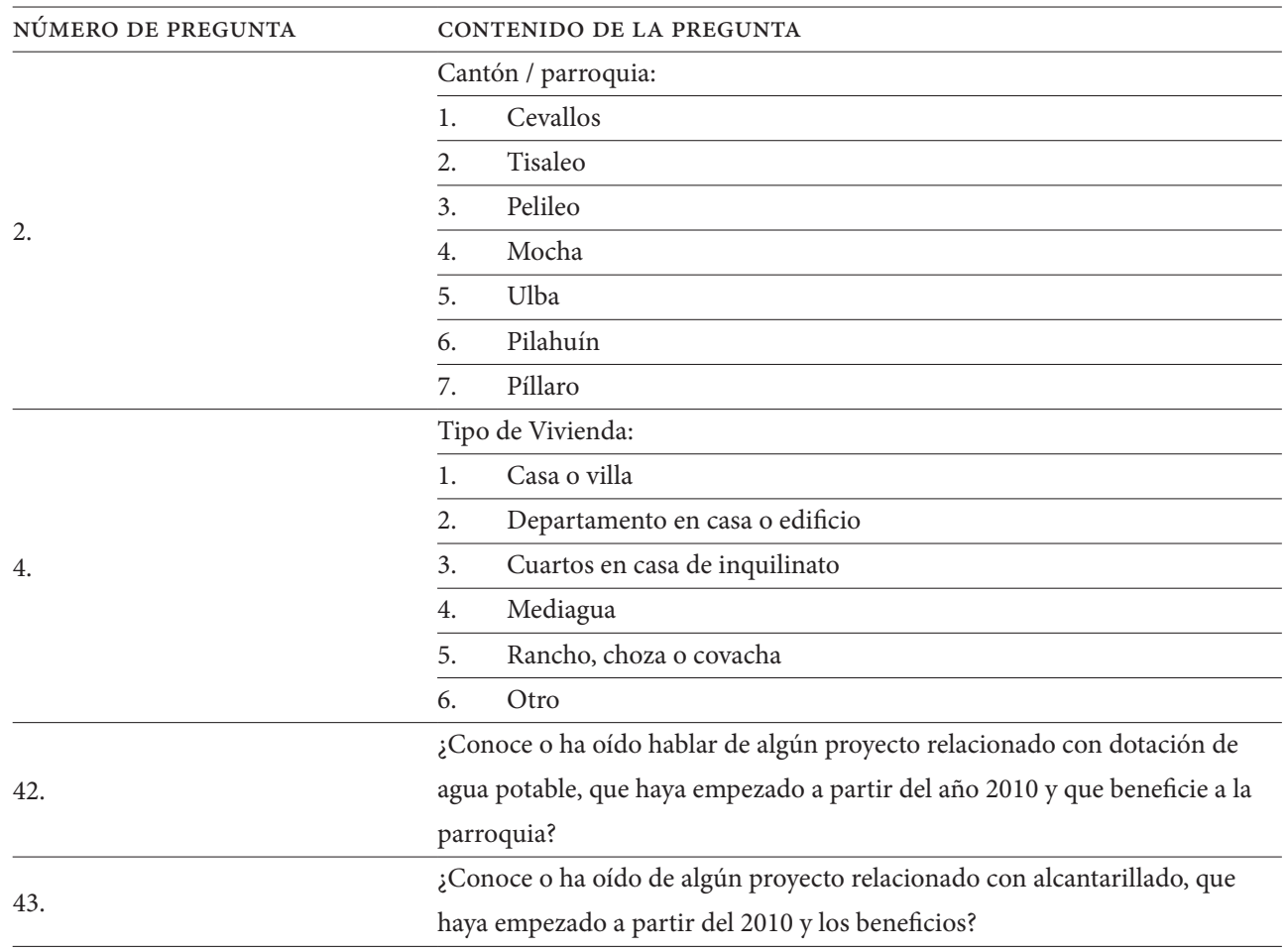

Fuente: autores (encuesta aplicada).

temporal que requieren estos sectores (Galvis y Meissel, 2009). Las soluciones al problema de la marginación de las periferias en el desarrollo socioeconómico promueven políticas efectivas para cambiar la perspectiva del Estado en cuanto a la inversión pública. La intensificación de la obra pública determina el desarrollo urbanístico de estas localidades, así como el compromiso fiscal de asignar recursos económicos al desarrollo de las comunidades de las periferias.

\section{ANÁLISIS ECONOMÉTRICO: MODELO SANOVA Y ANOVA}

Para este estudio se propone un análisis econométrico SANOva que medirá el grado de diferencia de las variables planteadas cuando éstas son sometidas a diferentes sistemas dependiendo de su zona geográfica. Las variables que se contrastan en este trabajo son: la calidad de vivienda en relación a la ubicación de los asentamientos urbanos de las residencias. El análisis ANOva permite verificar la relación entre la calidad de vida y la presencia de obras públicas en las localidades.

En primera instancia se optó por obtener los datos directamente del lugar de estudio, se realizó una encuesta dirigida a los pobladores de ocho localidades o asentamientos urbanos de la provincia de Tungurahua (Ecuador), en los cantones Cevallos, Tisaleo, Pelileo, Mocha, Píllaro; no se consideraron los cantones de Patate y Quero porque sus condiciones son similares a sus 
Tabla 2. Identificación de la variables para los modelos econométricos

\begin{tabular}{ll}
\hline VARIABLES & IDENTIFICACIÓN DE LAS VARIABLES \\
\hline Cantón / parroquia: & $\mathrm{X} 1$ \\
\hline Cevallos & $\mathrm{X} 2$ \\
\hline Tisaleo & $\mathrm{X} 3$ \\
\hline Pelileo & $\mathrm{X} 4$ \\
\hline Mocha & $\mathrm{X} 5$ \\
\hline Ulba & $\mathrm{X} 6$ \\
\hline Pilahuín & $\mathrm{X} 7$ \\
\hline Píllaro & $\mathrm{X} 8$ (variable de referencia) \\
\hline San Andrés de Píllaro & $\mathrm{X} 9$ \\
\hline $\begin{array}{l}\text { ¿Conoce o ha oído hablar de algún proyecto relacionado con } \\
\text { dotación de agua potable, que haya empezado a partir del año }\end{array}$ & \\
\begin{tabular}{l} 
2010 y que beneficie a la parroquia? \\
\hline Conoce o ha oído de algún proyecto relacionado con alcanta- \\
rillado, que haya empezado a partir del 2010 y los beneficios?
\end{tabular} & $\mathrm{X} 10$ \\
\hline Fuente: autores (encuesta aplicada). & \\
\hline
\end{tabular}

vecinos Pelileo y Cevallos, respectivamente, así como el cantón Baños, puesto que esta ciudad tiene características particulares en su proceso de desarrollo socioeconómico que merecen otro tipo de investigación. El análisis considera también las parroquias de Ulba, Pilahuín y San Andrés de Píllaro como nivel referencial.

El diseño se elaboró tomando en consideración una parroquia rural de la ciudad de Ambato, Pilahuín, bajo las premisas de distancia y población, en el estudio se identificó que es la parroquia más alejada y con una población representativa. Para tal efecto se realizó una encuesta a 383 habitantes, dato obtenido a partir de la fórmula para el cálculo del tamaño de la muestra y con una población de los cantones en estudio de la provincia de Tungurahua, que para el caso es de 153.340, estas encuestas fueron porcentualmente distribuidas de acuerdo a la cantidad de habitantes de cada uno de ellos. El estudio prevé un nivel de confianza de 95\%, probabilidad de éxito y fracaso de 50\% respectivamente y un nivel de significancia del 5\%.

El cuestionario realizado constó de cincuenta preguntas tomadas de la encuesta de condiciones de vida del Instituto Nacional de Estadísticas y Censos (INEC) (ver Tabla 1). Con las preguntas anteriormente mencionadas, se realizaron los análisis SANOVA y ANOvA propuestos en este artículo. Toda la metodología econométrica será realizada con la utilización del software econométrico Gretl. Para la depuración de los datos se procedió a clasificar las variables y numerarlas para que puedan ser operacionales en el software. Para la variable calidad de vida, fue necesario clasificar cada una de las categorías del cuestionario en forma ascendente (ver Tabla 2).

Para realizar el análisis SANOva se procedió a clasificar numéricamente la ubicación del sector como 1 para quienes habitan en un determinado lugar y o para quienes residen en un lugar diferente; teniendo en cuenta que los modelos ANOva son utilizados para establecer el nivel de significación estadística de la relación entre una regresada cuantitativa y regresoras cualitativas o dicótomas. Por lo general se emplean para comparar las diferencias entre los valores medios 
Tabla 3. Análisis de la regresión

\begin{tabular}{|c|c|c|c|}
\hline \multicolumn{4}{|l|}{ MODELO SANOVA } \\
\hline VARIABLES & COEFICIENTE & ESTADÍSTICO T & VALOR P \\
\hline Const. & 5,08333 & 39,89 & $5,44 \mathrm{E}-137$ \\
\hline$\overline{X 1}$ & $-0,22619$ & $-1,153$ & 0,2498 \\
\hline $\mathrm{X} 2$ & $-1,04078$ & $-5,745$ & $1,91 \mathrm{E}-08$ \\
\hline $\mathrm{X} 3$ & $-2,6067$ & -17 & $1,94 \mathrm{E}-48$ \\
\hline $\mathrm{X} 4$ & $-2,875$ & $-13,03$ & $2,97 \mathrm{E}-32$ \\
\hline $\mathrm{X} 5$ & $-2,53788$ & $-8,599$ & $2,21 \mathrm{E}-16$ \\
\hline $\mathrm{X} 6$ & $-3,6305$ & $-20,64$ & $9,11 \mathrm{E}-64$ \\
\hline $\mathrm{X} 7$ & $-0,117816$ & $-0,6839$ & 0,4945 \\
\hline $\mathrm{R}$ - cuadrado & 0,707122 & R - cuadrado ajustado & 0,701655 \\
\hline Valor p (de Fisher) & $6,32 \mathrm{E}-96$ & & \\
\hline \multicolumn{4}{|c|}{ ANÁLISIS DE VARIANZAS CALIDAD DE VIVIENDA } \\
\hline Cantón & Fisher & & \\
\hline Cevallos & \multicolumn{3}{|c|}{$\mathrm{F}(1,381)=32,3362($ Valor p $2,58 \mathrm{e}-008)$} \\
\hline Tisaleo & \multicolumn{3}{|c|}{$\mathrm{F}(1,381)=7,74266($ Valor p 0,0057$)$} \\
\hline Pelileo & \multicolumn{3}{|c|}{$\mathrm{F}(1,381)=60,0698($ Valor p 8,41e-014) } \\
\hline Mocha & \multicolumn{3}{|c|}{$\mathrm{F}(1,381)=15,2571($ Valor p 0,0001$)$} \\
\hline Píllaro & \multicolumn{3}{|c|}{$\mathrm{F}(1,381)=72,9791($ Valor p 3,19e-016) } \\
\hline \multicolumn{4}{|l|}{ Parroquias } \\
\hline Ulba & \multicolumn{3}{|c|}{$\mathrm{F}(1,381)=3,43966($ Valor p 0,0644$)$} \\
\hline Pilahuín & \multicolumn{3}{|c|}{$\mathrm{F}(1,381)=121,484($ Valor p $1,04 \mathrm{e}-024)$} \\
\hline \multicolumn{4}{|l|}{ MODELO ANOVA } \\
\hline VARIABLES & COEFICIENTE & ESTADÍSTICO T & VALOR P \\
\hline Const. & 2,11349 & 36,09 & $1,09 \mathrm{E}-124$ \\
\hline X9 & 2,47176 & 12,43 & $5,59 \mathrm{E}-30$ \\
\hline $\mathrm{X} 10$ & 0,349854 & 1,761 & 0,079 \\
\hline $\mathrm{R}$ - cuadrado & 0,743189 & $\mathrm{R}$ - cuadrado ajustado & 0,741833 \\
\hline Valor p (de Fisher) & $1,30 \mathrm{E}-112$ & & \\
\hline \multicolumn{4}{|c|}{ ANÁLISIS DE VARIANZAS CALIDAD DE VIDA } \\
\hline \multicolumn{4}{|l|}{$\begin{array}{l}\text { Obras públicas de agua } \\
\text { potable y alcantarillado }\end{array}$} \\
\hline \multicolumn{4}{|l|}{ Variable de referencia } \\
\hline San Andrés & \multicolumn{3}{|c|}{$\mathrm{F}(1,381)=67,0667($ Valor p $4,01 \mathrm{e}-015)$} \\
\hline
\end{tabular}

de varias categorías. (Damodar y Porter, 2009). Para la presente investigación, esta definición del modelo es importante porque permite tratar las variables cualitativas como distancia, calidad de vivienda y calidad de vida.

Para el análisis SANOva se procederá a realizar el análisis de regresión entre la calidad de vivienda como regresada y los asentamientos urbanos descritos anteriormente como 
regresoras que serán estudiadas econométricamente a través de mínimos cuadrados ordinarios. Posteriormente se realizará una observación individual de las diferencias de las varianzas de cada una de las relaciones de la regresada con las variables espaciales o asentamientos urbanos. Para el análisis de la respuesta con respecto a los estímulos: obras públicas de agua potable (x9) y obras públicas de alcantarillado (x10) se realizará un análisis ANOva tradicional, es decir, no espacial a través de una regresión múltiple con mínimos cuadrados ordinarios y con el análisis individual de las diferencias de las varianzas. Finalmente, se realizará una predeterminación de cómo se comporta la relación entre la calidad de vivienda y la distancia que tienen los asentamientos urbanos con la cabecera cantonal de la provincia de Tungurahua. Para la estimación del modelo SANOVA y ANOvA de regresión se presenta la categorización numérica realizada para las variables dependientes e independientes.

\section{RESULTADOS}

\section{ANÁLISIS SANOVA DE LA CALIDAD DE VIVIENDA VERSUS UBICACIÓN}

En primera instancia se procederá a realizar el análisis SANOva a través de mínimos cuadrados ordinarios para poder interpretar la significación de los parámetros y establecer las relaciones entre las variables implicadas. Se puede observar que 6 parámetros, incluida la constante, son estadísticamente significativos (ver Tabla 3), el estadístico de Fisher muestra un valor de probabilidad de apenas $6,32 \times 10^{-96} \mathrm{y}$ el coeficiente de determinación ajustado presenta un valor de 0,7017 ; lo que representa que los estimadores son insesgados y eficientes, variables independientes explican en un $70,17 \%$ a la variable dependiente.

En la misma tabla se puede observar que para el cantón Cevallos (x1), existe una disminución de o,22 puntos en la calidad de la vivienda, que no es un dato significativo ya que su $p$ valor es mayor que el nivel de significancia de 0,05. Para el cantón Píllaro (x7) se estima una disminución en promedio de la calidad de vivienda en apenas o,11 puntos, que no es un dato significativo ya que su $p$ valor es mayor que el nivel de significancia de 0,05. Es importante mencionar que se estimó para la parroquia de San Andrés de Píllaro un nivel de calidad de vivienda de 5, debido a que este lugar está tomado como el nivel de referencia en el estudio, es decir, que en promedio los habitantes de este lugar viven en departamentos, en casas o edificios.

\section{ANÁLISIS SANOVA A TRAVÉS DE LAS DIFERENCIAS DE LAS VARIANZAS}

Para llevar a cabo el análisis SANOvA es necesario realizarlo a través del estudio de cada una de las relaciones individuales entre las regresoras y la regresada, por lo que en este apartado se describirá la significación estadística de las diferencias de las varianzas de la regresada con respecto a sus variables independientes. Se puede observar que las diferencias de las varianzas de la relación calidad de la vivienda referente a los cantones y parroquias de este estudio, son significativos, ya que los valores $p$ del estadístico de Fisher son menores que el nivel de significancia de 0,05 . 


\section{ANÁLISIS ANOVA DE LA CALIDAD DE VIDA VERSUS OBRA PÚBLICA}

En cuanto al análisis de la relación entre la calidad de la vida y la obra pública a través de los proyectos de agua potable y alcantarillado que se ejecutan en las localidades estudiadas - se estableció una relación entre la calidad de vida como variable dependiente y proyectos de alcantarillado y de agua potable como independientes-, el estimador de la variable obras públicas de agua potable (x9) es estadísticamente significativo, mientras que el estimador de la variable obras públicas de alcantarillado (x10) es poco significativo, lo cual determina que tanto las obras de agua potable como de alcantarillado inciden en el promedio del nivel de la calidad de vida de la población. Por otro lado, el valor de probabilidad del estadístico de Fisher de $1,3 \times 10^{-12}$ demuestra que el modelo es insesgado y eficiente en su conjunto y un coeficiente de determinación ajustado de o,7418 indica que las variables independientes explican en un $74,18 \%$ a la variable dependiente.

Las diferencias de las varianzas de la relación entre la calidad de la vida y las obras públicas de agua potable y alcantarillado son estadísticamente significativas, ya que se observa un valor de probabilidad del estadístico de Fisher de 1,5 $\times 10^{-13}$.

\section{CONCLUSIONES}

En el análisis de heterogeneidad espacial a través del estudio sAnova, se pudo observar que las variables de ubicación x2, x3, x4, x 5 y x6, es decir, las correspondientes a los cantones Tisaleo, Pelileo, Mocha y las parroquias Ulba y Pilahuín, poseen estimadores estadísticamente significativos lo que quiere decir que la ubicación sí influye en la calidad de vivienda. Además, conforme la ubicación se va distanciando de la cabecera provincial Ambato, la disminución de la calidad de vivienda se va incrementando, es así que para el cantón Cevallos, que se ubica apenas a $14 \mathrm{~km}$ de la ciudad de Ambato, la disminución en promedio del nivel de la calidad de vivienda es de 0,22 puntos, mientras que para la parroquia de Pilahuín, que se encuentra ubicada a $20 \mathrm{~km}$ de la citada ciudad, se observa una disminución en promedio de 3,63 puntos en el nivel de calidad de la vivienda. La calidad de vivienda en las ciudades de Cevallos y Píllaro no se ven influenciadas por la distancia, dado que sus estimadores SANOva no fueron estadísticamente significativos; esto se debe a que estos cantones han logrado desarrollarse de manera independiente a la cabecera provincial, logrando desarrollar actividades económicas basadas en el turismo y en el comercio de artesanías autóctonas de la zona.

Por otro lado, a través del modelo ANOva — de la relación entre la variable calidad de vida con respecto a la presencia de obra pública-, se determinó que existe una fuerte influencia de esta última en el desarrollo de la calidad de vida, independientemente de la ubicación en la que se encuentre la residencia. Por consiguiente, dado que los estimadores de obras públicas de agua potable y alcantarillado son estadísticamente significativos, tanto individualmente como en su conjunto, se concluye que la inversión pública en proyectos de infraestructura interviene en el desarrollo de la calidad de vida de los residentes de la provincia. El valor del suelo tiende a incrementarse conforme se presentan proyectos de infraestructura de los gobiernos locales, lo cual implica un incremento de la plusvalía de los bienes inmuebles beneficiados por la presencia de obra pública, esto supone que los residentes realizan mejoras en sus 
viviendas conforme se mejora la calidad de los servicios públicos. Como recomendación, a partir de lo expuesto se recomienda profundizar en los estudios de econometría espacial utilizando, a más de las herramientas propuestas, otras que mejoren los resultados y que provoquen la discusión sustantiva en el área geoespacial.

\section{REFERENCIAS}

Chasco, C. (2001). Modelos de heterogeneidad espacial. Universidad Autónoma de Madrid, (s. d.), 68-102. Colli, B. (1981). Diccionario económico y financiero. Madrid, España: Arroyomolinos Mostoles.

Damodar, G. y Porter, D. (2009). Econometría. México DF, México: McGraw-Hill Interamericana.

Ferrada, L. y Zarzosa, P. (2010). Diferencias regionales en la participación laboral femenina en Chile. Cuadernos de Economía, 252, (s. d.).

Fitch, J., Soto, K. y Garza, R. (2013). Valuación de la calidad urbano-ambiental. Una modelación hedónica: San Nicolás de los Garza, México. Estudios demográficos y urbanos, (s. d.), 384-428.

Galvis, L. y Meissel, A. (2009). Fondo de Compensación Regional: Igualdad de oportunidades para la periferia colombiana. Economía y Sociedad, (s. d.), 50-70.

Guevara, T. (2013). La renovación como estrategia de desarrollo urbano en Buenos Aires (1996-2011). Apuntes, (s. d.), 78-79.

Mena, C., Morales, Y., Gajardo, J. y Ormazábal, Y. (2007). Análisis geoespacial de servicios básicos para las viviendas de Longaví, Retiro and Parral, Maule Region. Panorama socioeconómico, (s. d.), 106-116. 